\title{
Téoros
}

Revue de recherche en tourisme

\section{Tourisme international et tiers monde : l'enjeu des devises}

\section{René Baretje}

Volume 7, numéro 3, novembre 1988

Économie du tourisme

URI : https://id.erudit.org/iderudit/1080378ar

DOI : https://doi.org/10.7202/1080378ar

Aller au sommaire du numéro

Éditeur(s)

Université du Québec à Montréal

ISSN

0712-8657 (imprimé)

1923-2705 (numérique)

Découvrir la revue

Citer cet article

Baretje, R. (1988). Tourisme international et tiers monde : l'enjeu des devises.

Téoros, 7(3), 10-14. https://doi.org/10.7202/1080378ar d'utilisation que vous pouvez consulter en ligne.

https://apropos.erudit.org/fr/usagers/politique-dutilisation/ 


\section{Tourisme international et tiers monde I'enjeu des devises}

\begin{abstract}
"There are yet some other petty things which seem to have reference to this ballance of which the said officers of His Majesties Customs can take to notice, to bring them into account: as namely the expenses of travelers".

\section{Thomas Munn, England's Treasure} by Foreign Trade, 1620
\end{abstract}

Plus de trois siecles et demi plus tard, les dépenses des touristes ne peuvent plus être considérées comme des petty things.

Quelques années après la fin de la seconde guerre mondiale, de nombreux pays, essentiellement de l'Europe Occidentale, ont joué à fond la carte du tourisme en général et du tourisme international en particulier. Ces pays, dans la phase de reconstruction et de relance de la croissance économique, étaient à la recherche des devises indispensables au redémarrage de leur économie, et dès lors que la théorie du foreign exchange gain était toujours présente à l'esprit des décideurs, ces derniers ont considéré tout naturellement le tourisme comme l'un des pourvoyeurs essentiels de devises. Et alors de consacrer et d'orienter les moyens financiers vers la réalisation d'aménagements, d'équipements, d'installations destinés en priorité à attirer la clientèle internationale.

Cette idée a fait progressivement son chemin et, devant le succès rencontré par certains pays occidentaux, elle a été relayée et amplifiée par des instances internationales: U.I.O.O.T., Nations Unies, CNUCED, OCEE, UNESCO, Banque Mondiale, etc.

Ces instances ont, dans un premier temps. misé à fond sur l'importance économique du tourisme international (le tourisme apporte des devises, le tourisme crée des emplois, le tourisme assure un développement régional équilibré...) et ont vivement recommandé ensuite à tous les pays développés et en voie de développement, capitalistes et socialistes, de sé lancer à corps perdu dans l'exploitation de leurs ressources touristiques, assurés qu'ils seraient en fin de compte de récupérer la manne tant espérée, les devises.

Cette promotion subite et tous azimuths $s$ 'est étalée sur près de deux décennies et s'est traduite par une course effreinée entre les pays des différents continents, décidés à capter la clientèle internationale de plus en plus nombreuse.

- Directeur, Centre des Hautes Etudes Touristiques, Aix. en-Provence, France.
Cette promotion était néanmoins hấtive et ne reposait malheureusement pas sur une connaissance exacte des mécanismes économiques qui président au fonctionnement de l'industrie touristique.

Certains pays qui s'étaient mis sur les rangs, alléchés par les promesses de rentabilité, se sont progressivement rendus compte que les effets économiques tant attendus étaient loin de se manifester; et ces mémes pays, voire d'autres, ont découver également et progressivement que la pratique du tourisme international non seulement pouvait provoquer des effets économiques négatifs, mais également entrainer des perturbations très graves sur le plan social, culturel, écologique, politique...

Les réserves de plus en plus nombreuses, assorties souvent de critiques très acerbes, voire de remises en cause, ont à leur tour fait leur chemin et provoqué un renversement de la tendance dans la doctrine des instances internationales ${ }^{(1)}$.

Ce changement de cap s'est opéré précisément à une période chamière qui coinncide avec l'apparition de la crise énergétique de 1973-1974 et la cohorte de ses répercussions. Cette coincidence, si coincidence il y a, n'a fait que précipiter la position des instances internationales et particulièrement celle de 1'UIOOT/OMT ${ }^{2}$ '.

La Déclaration Finale de la Conférence de Manille met bien en relief la primauté accordée dorénavant et sans ambiguité au tourisme national par rapport au tourisme international, et la primauté accordée dorénavant et sans ambiguïté aux aspects sociaux, cultu= rels et éducatifs sur les aspects économiques. Le tourisme international n'est pas pour autant condamne, mais compte tenu des liens de dépendance accrue qu'il a entraînés entre pays développés-émetteurs et pays en voie de développement-récepteurs'si les autorités internationales, de concert, recommandent dorénavant une approche réaliste qui consiste à évaluer d'une manière objective l'apport net en devises. Elles préconisent l'étalissement d'une véritable comptabilité du tourisme international; elles indiquent quelles opérations il faut prendre en considération, sans proposer pour autant un véritable outil de gestion opérationnel permettant de dégager l'apport net en devises.

A défaut donc de s'attaquer à l'épineux problème de la mesure de l'apport net en devises par le tourisme international, toutes les institutions publiques chargées de dresser un bilan monétaire du tourisme international se contentent, aujourd hui encore de ne recenser que les seules dépenses des touristes une fois rendus à l'étranger; elles n'appréhendent donc que l'apport brut en devises.

Deux méthodes principales sont utilisếes séparément ou conjointement pour cerner lo montant des devises laissées par les touristes dans un pays étranger:

- la méthode directe, ou méthode des flux financiers, ou méthode de la Banque Centrale, basée sur les informations fournies par les touristes auprès des établissements bancaires et assimilées et regroupées par les banques centrales et leurs offices de change ou organismes similaires.

- la méthode indirecte, ou méthode des flux physiques, ou méthode du Ministère du Tourisme, qui consiste à établir le montant global des dépenses en multipliant le nombre de nuitées des étrangers par un montant moyen des dépenses journalières.

Il faut bien convenir que ces deux procédés sont loin de saisir l'ensemble des transactions commerciales liées aux dépenses réelles des touristes $^{(4)}$. Malgré toutes les réserves formulées à l'encontre des méthodes utilisées pour dégager le solde brut de la balance touristique, il faut bien convenir que tous les pays sont contraints de pratiquer alternativement ou conjointement l'une ou l'autre de ces méthodes restrictives.

Sur la base de ces informations, les pays se livrent alors à des traitements traditionnels pour tenter de positionner la rubrique tourisme par rapport à d'autres éléments de la balance des paiements courants (voire d'autres paramètres: produit intérieur brut. consommation finale privée des ménages, ...) ou particulier pour déterminer l'influence de la rubrique tourisme sur le solde de la balance des marchandises et services amputé du tourisme $e^{(5)}$.

Mais si l'on veut connaitre réellement la situation nette des échanges touristiques internationaux, il faut bien se décider à abandonner la définition étroite du Fonds Monétaire International ou celle des Nations Unies qui ne prennent en consideration que les seules dépenses des touristes une fois rendus à l'étranger. La balance touristique traditionnelle ne fournit qu'une vue fragmentaire des transactions monétaires engendrées par le déplacement des personnes. La satisfaction des besoins de la clientèle exige en effet la 

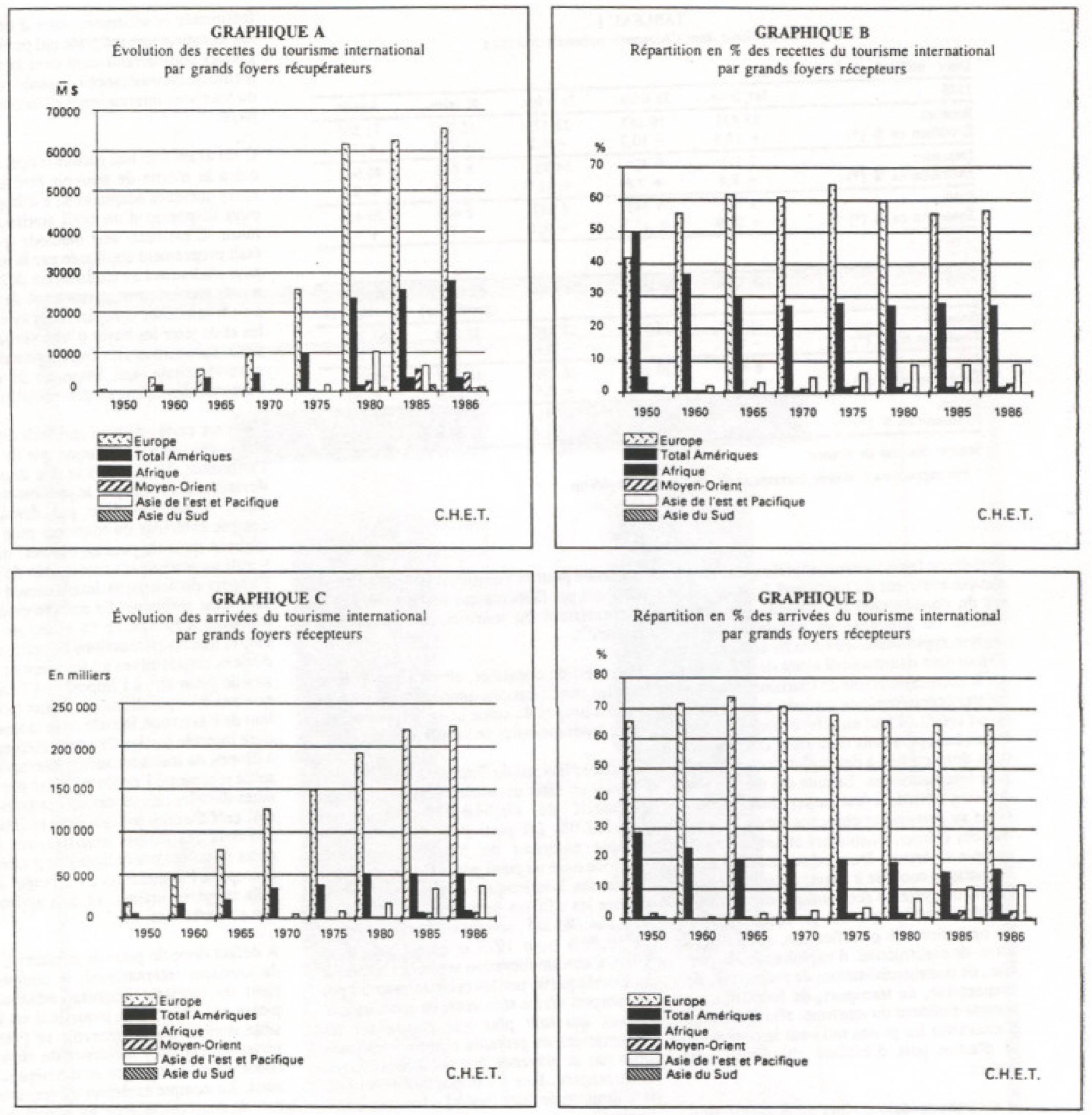

prise en compte d'un certain nombre d'éléments directement lies au tourisme et comptabilisés dans les autres rubriques de la balance des paiements: mouvements divers de capitaux, coúts de transport, services divers, exportations et importations de marchandises et de biens d'équipement, etc.

Un compte d'exploitation touristique, qui regrouperait toutes les charges et tous les produits permettrait seul de dégager la véritable nature du solde. Tout laisse à penser que ce solde serait loin d'être identique à celui qui apparait actuellement dans la balance des paiements du FMI. Des renversements de situation seraient à attendre.

La dépendance vis-à-vis de l'étranger en matière de biens, de services de capitaux, ou le fait d'être une nation complexe modifie le sens et le montant du solde des transactions touristiques. Si le solde de la balance des paiements ne doit pas changer, sa structure s"en trouvera altérée, du fait que certaines opérations enregistrées tant au débit qu'au crédit de multiples rubriques y seraient retirées pour être réparties au nouveau compte d'exploitation.
Conséquence inévitable de cette nouvelle imputation, tous les scores touristiques proclamés jusqu'à ce jour seraient remis en cause. En effet,

- tel pays connaîtrait un changement du signe algebrique de sa balance touristique;

- ou verrait son solde positif s'améliorer;

- ou enregistrerait au contraire une stabilité relative de son solde créditeur ou débiteur:

- ou devrait s'atiendre à voir son solde déja négatif s'aggraver. 
TABL.EAU 1

Le tourisme dans l'economie nationale française

Unitt: milliards de francs courants

\begin{tabular}{lccccc}
\hline 1985 & ler trim. & 2e trim. & 3e trim. & 4e trim. & Anné \\
\hline Receties & 13435 & 19167 & 23477 & 15277 & 71356 \\
Evolution en $\%\left(^{*}\right)$ & $+17,6$ & $+10,7$ & $+4,7$ & $-0,5$ & $+7,3$ \\
\hline Depenses & 7715 & 9424 & 14935 & 8868 & 40942 \\
Evolution en $\%\left(^{*}\right)$ & $+8,4$ & $+7,6$ & $+12,8$ & $+7,3$ & $+9,6$ \\
\hline Soldes & 5720 & 9743 & 8542 & 6409 & 30414 \\
Evolution en $\%\left(^{*}\right)$ & $+32,9$ & $+13,7$ & $-7,1$ & $-9,7$ & $+4,4$ \\
\hline
\end{tabular}

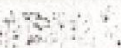

\begin{tabular}{lccccc}
\hline 1986 & Ier trim. & 2e trim. & 3e trim. & $\begin{array}{c}\text { 4e trim. } \\
\text { (provisoire) }\end{array}$ & $\begin{array}{c}\text { Anné } \\
\text { (provisoire) }\end{array}$ \\
\hline Receties & 12329 & 17966 & 22880 & 14040 & 67215 \\
Evolution en $8(*)$ & $-8,2$ & $-6,3$ & $-2,6$ & $-8,1$ & $-5,2$ \\
\hline Dépenses & 8439 & 10164 & 16199 & 10248 & 45050 \\
Évolution en $\%(*)$ & $+9,4$ & $+7,8$ & $+8,5$ & $+15,6$ & +10 \\
\hline Soldes & 3890 & 7802 & 6681 & 3792 & 22165 \\
Evolution en $\%(*)$ & $+32,0$ & -20 & $-21,8$ & $-40,9$ & $-27,2$ \\
\hline
\end{tabular}

Source: Banque de France

- Par rappor au trimestre correspondant de l'année précédente

Peut-on en fait concevoir et réaliser un pareil compte extérieur du tourisme? Peut-on passer du conditionnel au présent? Oui, sans réserve de procéder au préalable à une analyse approfondie du système économique du tourisme international avant de déboucher sur le compte extérieur du tourisme. Pour alimenter précisément ce compte, tant au débit qu'au crédit, il faut en effet d'abord repérer toutes les opérations traduisant des transactions donnant lieu à des règlements ou transferts internationaux. Ensuite qui dit compte, dit comptabilité; il faut passer alors du théorique au pratique et chercher à évaluer chacun des eléments débiteurs et créditeurs du compte extérieur. Dans ce contexte, la problématique consiste à passer du schéma descriptif du système économique du tourisme international ${ }^{(6)}$ - prenant en compte toutes les opérations de planification, d'aménagement, de construction, d'exploitation, de gestion, de commercialisation, de promotion, de francement, de transport, de formation au compte extérieur du tourisme, afin d"une par d'enumérer les postes quil vont le composer et d'autre part d'évaluer chacun de ces postes.

A maintes occasions, lors de la description du système économique du tourisme international, apparaissent un certain nombre d'operations et de transactions donnant lieu à des transferts de devises au comptant ou à terme. Il reste alors à repérer puis à regrouper les transactions de meme nature et enfin a les inscrire dans les rubriques correspondantes, au débit et au crédit, du compte extérieur du tourisme. Ce travail de recollement aboutit au tableau no 2 où les numéros affectés à chaque rubrique, débitrice ou créditrice correspondent à des situations décrites précédemment lors de l'analyse du système economique.

Une illustration chiffrée (tableau 3) peut étre proposée pour la Tunisie en 1977 et en 1980 , attestant par là même que le modèle du compte extérieur du tourisme est bien opérationnel ${ }^{7}$.

Force est de constater, au vu des résultats, que les modifications annoncées précédemment à propos du solde de la balance touristique traditionnelle, se vérifient amplement.

L'Office National du Tourisme Tunisien proclamait en effet un solde brut d'un montant respectif de 80.348 .975 DT et de 134.237 .031 DT pour 1977 et 1980 . Or, le compte extérieur du tourisme, selon la méthode mise au point par le Centre des Hautes Études Touristiques d'Aix-en-Provence, avance les chiffres de 88.313 .002 DT et de 168.154.645 DT, soit un solde net supérieur de $10,99 \%$ pour 1977 et de $12,52 \%$ pour 1980. Cette amélioration sensible s"explique en grande partie par les recettes induites par le transport aérien et la vente de produits artisanaux qui font plus que compenser les importations de produits alimentaires justifiées par la présence des nombreux touristes étrangers, face à l'impossibilité du secteur tunisien de faire face a la demande additionnelle.

Après avoir donc analysé la tuyauterie complexe du système touristique retraçant l'ensemble des échanges économiques qu'engendre dans une collectivité la prise en charge du phénomène touristique avant, pendant et après le séjour des excursionnistes, des week-enediers et des touristes, on est arrivé, après sélection des transactions donnant lieu à des transferts de devises, a présenter la structure du compte extérieur de la Tunisie.

Cette nouvelle façon de concevoir d'une manière globale les échanges touristiques internationaux est certes appelée à étre per- fectionnée et affirmée; mais d'ores et déjă elle constitue une méthode qui permet de progresser considérablement dans la vole d'une meilleure connaissance comptable de l'apport du tourisme international à l'économie d'un pays.

C'est avant tout une méthode opérationnelle qui a le mérite de pouvoir être appliquée, après quelques adaptations, à n'importe quel pays disposant d'un outil statistique minimum. C'est aussi une méthode qui, si elle était proprement appliquée par la totalité des pays s'adonnant à I'exploitation de leurs gisements touristiques, permettrait de procéder à de fructueuses comparaisons internationales et de jeter les bases d'une véritable politique communautaire interrégionale et (ou) intra-rćgionale, qui laisserait de côté tout l'arsenal des mesures protectionnistes.

Ce n'est certes qu'une méthode comptable amélioréc qui n'ambitionne que de recenser l'ensemble des recettes et des dépenses en devises engendrées par le phénomène touristique. Il ne faut donc pas demander au compte extérieur du tourisme plus d'informations qu'il ne peut en donner, ni surtout $y$ voir un instrument susceptible de mesurer I'impact du tourisme international sur une économie nationale. Le compte extérieur du tourisme n'enregistre en effet, au premier degré, que les transactions débitrices ou créditrices consécutives au tourisme et aux loisirs de plein air, à l'import et à l'export, et non pas les opérations en chaine qui découlent de l'insertion initiale de la masse monétaire injectée par les flux touristiques; ainsi, à propos du tourisme aérien international, le solde représente l'excédent laissé par les touristes dans les caisses des compagnies acriennes: cela n'exclut pas que pour satisfaire précisément ces mẻmes touristes, ces compagnies ne soient pas obligées de procéder par exemple à l'acquisition à l'étranger d'appareils supplémentaires, ni de s'approvisionner en carburant, etc

A défaut donc de pouvoir mesurer l'impact du tourisme international, le compte extérieur du tourisme constitue néanmoins la pierre angulaire, sans laquelle il est impossible dorénavant de pouvoir se prononcer sérieusement sur l'incidence du sérum tourisme dans les rouages économiques nationaux. Le compte extérieur du tourisme permet de mesurer la dose de sérum injectée; aux autres médecins de suivre les bienfaits ou les méfaits de la diffusion du sérum dans le corps économique.

Il faut bien convenir cependant que les pays qui se décideront à elaborer un pareil compte extérieur du tourisme verront le solde stricto sensu de la balance touristique subir de profondes modifications positives ou négatives.

Dans ce demier cas cependant, l'aggravation du solde de la balance du tourisme ne signifiera pas automatiquement la condamnation à priori de cette activité. Et là encore, le compte extérieur du tourisme va être d'un apport très précieux pour les responsables de la politique touristique; chaque poste du 
TABLEAU 2

Compie extérieur du tourisme

\begin{tabular}{|c|c|c|c|}
\hline Detpenses & Rubriques" & Recettes & Rubriques* \\
\hline Dépenses touristiques............................... & $\begin{array}{c}(17+19)= \\
29+31+33+35+37=79\end{array}$ & Recettes touristiques......... & $\begin{array}{c}(42+44)= \\
54+56+58+60+62=76\end{array}$ \\
\hline Transports & 68,71 & Transports & 70,71 \\
\hline 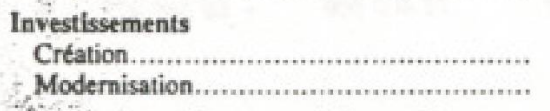 & $\begin{array}{c}98,99 \\
205,202\end{array}$ & 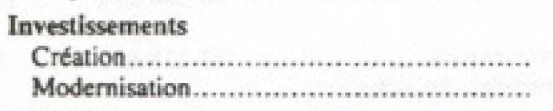 & $\begin{array}{c}89,90,91 \\
187,188,189\end{array}$ \\
\hline 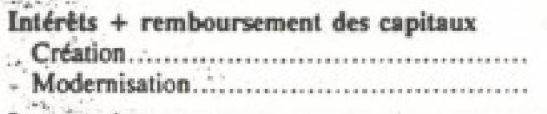 & $\begin{array}{l}158,160,161 \\
198,199,200\end{array}$ & 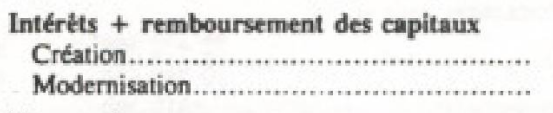 & $\begin{array}{l}167,168 \\
209,210\end{array}$ \\
\hline $\begin{array}{l}\text { Importations } \\
\text { Biens d'equipement............................... } \\
\text { Biens de consommation........................... }\end{array}$ & $\begin{array}{l}103,140,(143) \\
191\end{array}$ & $\begin{array}{l}\text { Exportations } \\
\text { Biens diequipement............................... } \\
\text { Biens de consommation............................. }\end{array}$ & 112,212 \\
\hline 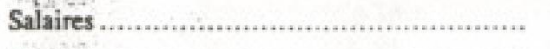 & $108,128,147,195,216$ & Salaires ...................... & 116,129 \\
\hline Formation .................................................. & 131 & 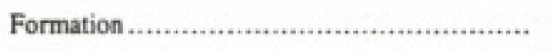 & 117.130 \\
\hline Profits & $171,172,173,174$ & Profits & $155,169,170$ \\
\hline Publicité ............... & $179,180,181$ & Publicitế ........................ & 157 \\
\hline 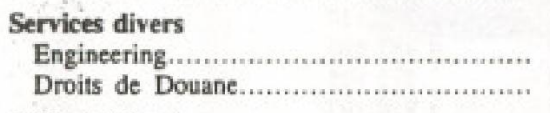 & $\begin{array}{c}218,219 \\
157\end{array}$ & $\begin{array}{l}\text { Services divers } \\
\text { Engineering......................................... } \\
\text { Droits de Douane }\end{array}$ & $\begin{array}{c}221,222 \\
157\end{array}$ \\
\hline Solde créditeur & & Solde débiteur & \\
\hline
\end{tabular}

- Voir notes explicatives no (4)

compte extérieur - surtout si ce dernier est établi sur deux ou plusieurs années - va constituer un clignotant ou une série de clignotants (un par rubrique) qui vont permettre de modifier les composantes du marché touristique et de réduire, à terme, la dépendance vis-à-vis de l'étranger.

L'État selon le cas, et pour accroitre ses recettes et (ou) diminuer ses dépenses, pourra alors conjointement ou successivement, mais en toute connaissance de causes:

- modifier sa politique de formation professionnelle:

- revoir le code des investissements et le régime fiscal touristique:

- réduire sa dépendance à l'égard des importations de biens d'équipement et de consommation par une diversification de ses productions;

- reconsidérer sa politique des transports;

- rechercher de nouveaux marchés:

- remettre en cause sa politique de distribution et de commercialisation:

- réexaminer sa stratégie de promotion et de publicité...

en d'autres termes, peser d"un poids différent sur telle ou telle rubrique du compte extérieur du tourisme.

D'aucuns ne manqueront pas de souligner que ramener la problématique économique du tourisme à un débat sur le foreign exchange gain. constitue une approche réductrice du phénomène et qu "on peut payer cet objectif - améliorer le solde de la balance touristique - d'un prix excessif. Il est en effet fréquent, pour garder la métaphore médicale utilisée plus haut, que la guérison d"une affectation particulière se paye par une dégradation partielle ou totale du système. Et il est évident que la thérapeutique touristique $a_{4}$ pour le corps social, culturel, économique, écologique, des effets analogues, encore qu'on souhaiterait voir la sociologie, l'économie et l'écologie se donner enfin la main pour élucider en priorité ce problème.

Pourtant d'un autre côté, il faut entrevoir combien cette réflexion est susceptible de désorienter le responsable politique ou economique si, après lui avoir recommandé de développer le tourisme pour améliorer sa balance des paiements, on le place quelques années plus tard devant le dilemne: garder le premier objectif, mais subir dans d'autres secteurs les effets négatifs inhérents à tel type de politique touristique, ou alors changer d'objectif, au prix de compensations dans d'autres secteurs, sans d'ailleurs qu'on puisse clairement quantifier les deux termes de l'alternative.

L'intérêt de la démarche tient, à condition d'aller plus loin, au fait qu'elle peut supprimer l'alternative et concilier les objectifs. Après avoir identifie les regrettables sideeffects du tourisme, comment imaginer une démarche qui garde tous les bénéfices et supprime ou minime ces side-effects?

On a donc essayé de mettre sur pied une démarche qui permet de ne pas modifier l'objectif en quelque sone historique du tourisme - I'acquisition de devises - mais qui permet egalement de prendre en compte I"articulation optimale des mesures indispensables concernant toutes les incidences du tourisme dans le domaine économique. social, culturel, éducatif, écologique.

Le compte extérieur du tourisme est d'abord un outil complable. Mais on voit du même coup que cette finalité peut être considérée comme accessoire. Le compte extérieur du tourisme peut et doit surtout devenir un outil de gestion, mais au prix de recherches complémentaires sur les impacts du tourisme et des loisirs de plein air sur les divers rouages économiques, sociaux, culturels, écologiques, afin de maîtriser quantitativement et qualitativement les variations induites par une variable du systeme sur les autres composantes de ce système; alors, et alors seulement, on pourrait savoir si on peut accroitre les résultats de telle ou telle rubrique du compte extérieur du tourisme, quelles sont les modifications qu'il va falloir introduire en amont et en aval du système pour atteindre les objectifs visés.

Du méme coup, apparaît la fonction majeure du compte extérieur du tourisme:

- un instrument de pilotage propre à déterminer les interventions diverses de l'État pour obtenir des effets sur des points déterminés comme objectifs prioritaires:

- un instrument de pilotage de la politique touristique du gouvernement permettant de mesurer non seulement les effets positifs sur les objectifs visés, mais aussi les effets négatifs jouant à contre-courant dans d'autres secteurs.

Objectifs, ou direction par objectifs, voilà le grand mot läché. Un compté extérieur du tourisme qui ne serait pas précedé d'une politique reposant sur la direction par objectifs. ne serail qu"un outil dépourvu d'utilité et qui plus est, l"adoption d'une pareille politique permettrait de concilier à la fois l"approche du tourisme national et l'approche du tourisme international, deux entités qui ne 
TABLEAU 3

Le comple extérieur du tourisme de la Tunisie - 1977-1980

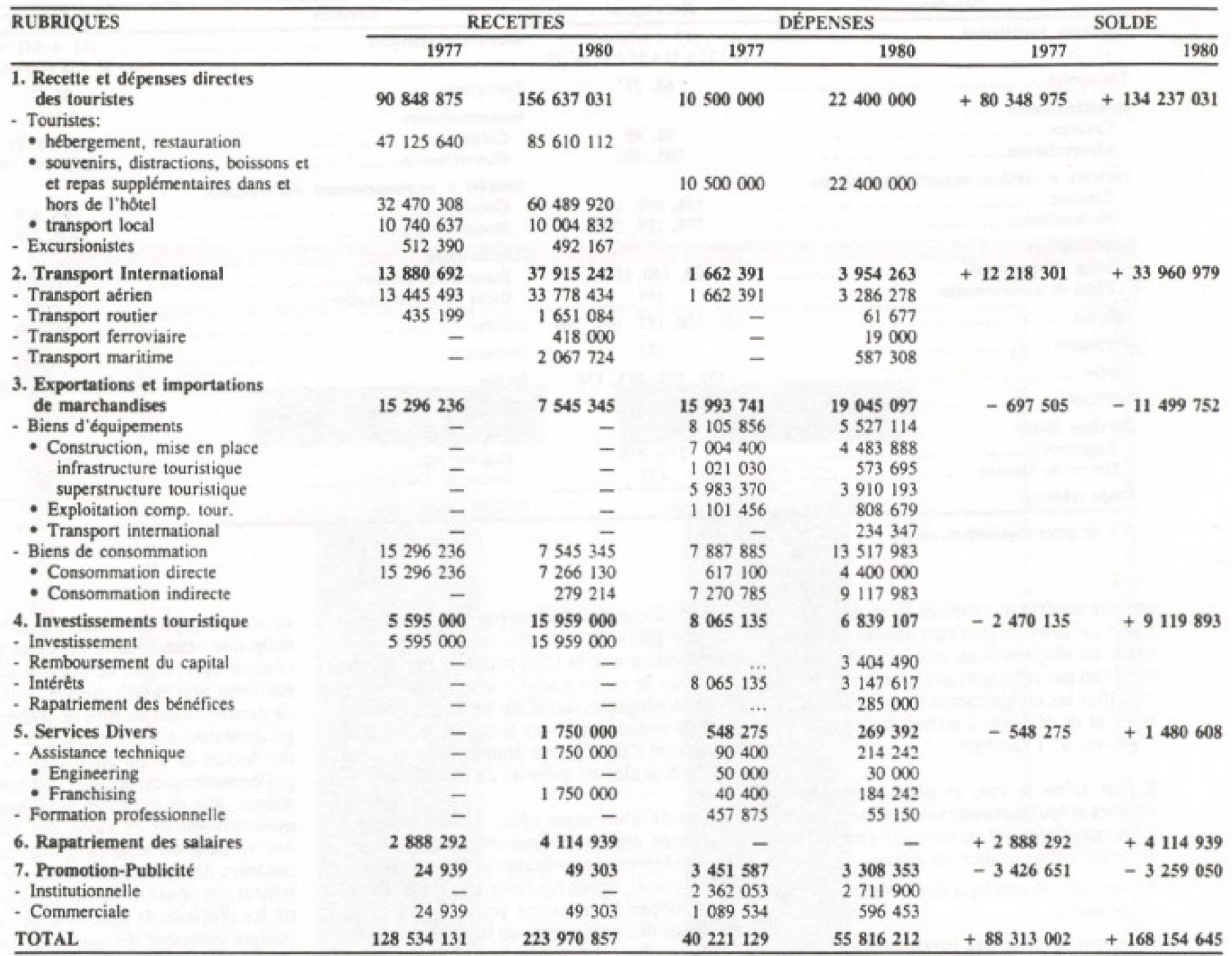

devraient plus être antagonistes mais complémentaires.

A l'examen du fonctionnement du système économique du tourisme et sur la base d'une observation dynamique de l'évolution du tourisme a l'échelle planétaire, force est de constater qu'il existe un équilibre très délicat et précaire entre le temps et le revenu qui ren= dent la demande touristique solvable, et l'espace que subit la charge exercée.

Sous la pression d'une multitude de facteurs, on assiste à de profondes mutations quantitatives et qualitatives qui font que, dorénavant, toutes les nations. quel que soit leur niveau de développement, doivent se préparer à affronter les problèmes de la civilisation des loisirs, en prenant en considération les besoins a la fois des autochtones et des étrangers, mais tout en conciliant les intéréts de la société, de l'économie et de l'environnement.

Le débat tourisme international ou tourisme national est un faux problème. Donner la priorité tantốt au tourisme national, tantôt au tourisme international est aussi un faux problème. Dès lors que le tourisme national et le tourisme international sont étroitement imbriqués et interdépendants, on ne peut concevoir simultanément deux politiques de tourisme menées parallèlement au niveau d'un même pays.

Encore faut-il se doter d'une politique adéquate, assortie d'objectifs hiérarchisés, de stratégies adaptées et de moyens appropriés pour rechercher l'équilibre harmonieux de la trilogie: SOCIÉTE/ECONOMIE/ENVIRONNEMENT. A ce prix, et à ce prix là seulement, on débouchera sur un Tourismé à haute Valeur Ajouté.

Notes explicatives

[1) Cf. R. Baretie, Contribution nette du tourisme international al la balence des paiements, OCDE, Comite Tourisme, Paris, mai 1984

(2) Union Internationale des Organismes Otficieis de Tourisme. devenue Organisation Mondiale du Tourisme.
(3) Sur le plan des investissements, des transports, des biens d'equipement et de consommation, de la distribution. de la main- d'oeuvre, de la promotionpublicite, de Pengineering, etc.

(4) $\mathrm{Cl}$. R. Baretje, Le compte exterieur du tourisme. CHET, Aik-en-Provence, Lets Cahiers du Tourisme. Serie C. No 46, juin 1978 .

(5) Sur lillustration de ces traitements, cf. R. Baretie, Tourisme et balance des paiements. Europe 1970-1980. Donntes OCDE. CHET, Aix-enProvence, Les Cahiers du Tourisme, Série D. No 5. novembre 1981. Tourisme et balance des paiements. Europe 1970-1979. Donneses F.M.I. CHET, Aix-en-Provence, Les Cahiers du Tourisme. Serie D, No 6, novembre 1981.

66) Pour la description et l'articulaiton des 222 parame. thes pris eñ consideration, cf. R. Baretje, Le compte extérieur du tourisme, op. cit.

17) Sur la presentation de tous les calculs intermediaires sur l'estimation des recertes et des depenses, of. C. Richter, Lè comple extérieur du tourisme de la Tunisie. Tourisme techanges internationaux dans les pays en voie de developpement. These. CHET, Aix-en-Provence, 1981. C. Richter, Le compte extérieur du tourisme de la Tunisie 1980. Office National du Tourisme Tunisien, Tunis, decem: bre 1982 Le Maroc. I'Algene en le Mexique font pré. sente-ment l'objet au CHET de recherches similaires.

14 TEOROS - Nal 7, no 3, navembere $19 \mathrm{geg}$ 